\title{
Stratégies de désignation dans le discours politique catholique et protestant pendant les guerres de religion : le tournant décisif de la conversion d'Henri IV (1593)
}

\author{
Sophie Yvert-Hamon (Université de Stockholm, Suède)
}

\begin{abstract}
The purpose of this paper is to analyze the strategies of designation in the political discourse of Huguenots on the one hand, and Ultra-Catholics on the other hand, during the period preceding and following the conversion of Henry IV (1593). Using Discourse Analysis as a theoretical and methodological framework, this study focuses on how the different actors (parties, the King) are presented in these discourses.

The corpus is composed of two texts, both published in 1593. The first one is by the Duke of Mayenne, leader of the Catholic League, and aims to reunify all Catholics within the kingdom in order to annihilate Protestantism. It is written before the conversion of Henry IV to Catholicism and expresses the frustration of Ultra-Catholics at having a protestant king. The second text is by Philippe Duplessis-Mornay on behalf of the Huguenots' political assemblies. It is a letter addressed to King Henry IV just after his conversion to Catholicism in 1593. This letter expresses the frustration of Huguenots as their protector converted to Catholicism. Analyzing the use of referential expressions according to the constructivist conception of the reference developed by Apothéloz and ReichlerBéguelin (1995), this study considers the referents as discourse-objects and the talking subject as acting on these objects. The study is qualitative and examines the different functions (argumentative, social, polyphonic) of the categorizations and recategorizations in order to underscore the discursive strategies of the authors. This paper argues that there are similarities in the way the different actors are presented in the two texts but that the perspective is essentially religious in the text by the Catholic League whereas the perspective is more political in the text by the Huguenots.
\end{abstract}

Keywords: Strategies of designation, political discourse, French Wars of Religion, conversion of Henry IV, Discourse Analysis.

\section{Introduction}

Se donnant pour cadre théorique et méthodologique l'analyse du discours, cette recherche porte sur les stratégies de désignation dans le discours politique des réformés d'une part et des ultra-catholiques d'autre part, au cours des années 1592 et 1593.

Dans une France déchirée par les guerres de religion opposant catholiques et protestants, modérés et extrémistes, la mort brutale d'Henri III, assassiné par le moine ligueur Jacques Clément, fait du protestant Henri de Navarre le nouveau roi de France. Non reconnu

ROM17 Edited by Jon Askeland, Marco Gargiulo and Synnøve Ones Rosales. 
par la majorité catholique, il doit conquérir son royaume. La question de la conversion du roi se pose de façon aiguë face aux résultats limités de la conquête militaire. Euvre des catholiques modérés, la conversion est un sujet de préoccupation tant pour les réformés que pour les ultra-catholiques de la Ligue.

Le corpus retenu pour cette étude est constitué d'un texte rédigé et publié par le duc de Mayenne, chef de la Ligue, peu avant la conversion du roi et d'un texte issu des assemblées politiques des protestants et rédigé peu après la conversion. Ces deux textes ont en commun d'exprimer la frustration et l'inquiétude autour de deux questions essentielles : la coexistence des catholiques et des protestants et la conversion du roi. Il s'agit donc de textes politiques émanant de groupes opposés, définis en partie par leur appartenance religieuse et cherchant à faire pression sur un tiers considéré comme essentiel sur l'échiquier politico-religieux. Ce tiers est incarné par les catholiques non ligueurs dans le texte de la Ligue et par le roi dans le texte protestant.

Nous intéressant à la façon dont les différents acteurs (le roi, les différents partis) sont mis en scène dans ces textes, nous formulons la double hypothèse suivante : nous supposons d'une part que ces discours opèrent une distinction entre la dignité royale et la personne incarnant cette dignité, et d'autre part que chacun des deux groupes tente de se présenter de façon homogène, effaçant les dissensions internes afin de recentrer la lutte sur une opposition binaire catholiques/protestants. Pour tester cette double hypothèse, cette étude se propose d'analyser l'emploi des expressions référentielles selon la conception constructiviste de la référence développée par Apothéloz et Reichler-Béguelin (1995), considérant les référents comme des objets-de-discours et le sujet parlant comme agissant sur ces objets. Les différentes fonctions (argumentative, sociale, polyphonique) des catégorisations et recatégorisations sont examinées afin de mettre en évidence les stratégies discursives des auteurs.

\section{Contexte historique}

Au cours de la seconde moitié du XVIème siècle, la France est le théâtre de guerres civiles opposant la majorité catholique et la minorité protestante ${ }^{1}$. Les périodes de guerre alternent avec des périodes d'accalmie encadrées par différents édits et paix. Chacun de ces deux groupes se subdivise selon plusieurs tendances, des modérés aux extrémistes. Sur le plan politique, trois partis se distinguent. Du côté protestant, l'Union calviniste est une organisation politique créée après les massacres de la Saint-Barthélemy, dotée d'un gouverneur général et protecteur des Églises réformées (Henri de Condé puis, à partir de 1576, Henri de Navarre). Elle est soutenue par l'Angleterre et certains princes germaniques notamment. Les catholiques sont divisés en deux grands groupes. La Ligue ou Sainte Union est le nom donné aux catholiques intransigeants organisés en union des catholiques français à partir de 1576 et animés par le désir d'extirper l'hérésie du royaume. Menée par les Guise, elle reçoit le soutien de l'Espagne catholique. Les catholiques modérés quant à eux, sont favorables à une politique de compromis et s'organisent en parti politique sous le règne d'Henri III. Appelés Politiques (le plus souvent par leurs adversaires), ils s'opposent de plus en plus clairement aux ligueurs. Animés par un sentiment national aigu et partisans du gallicanisme, ils voient d'un mauvais œil l'ingérence des puissances étrangères dans les affaires du royaume. La distinction entre la religion et l'État, motivée par la volonté de

\footnotetext{
${ }^{1}$ Voir notamment Jouanna et al. (1998).
} 
rétablir la paix intérieure, prend forme dans leur discours. Etienne Pasquier, avocat général à la chambre des comptes de 1585 à 1604, résume ainsi le paysage politico-religieux de la France au cours des dernières décennies du XVIème siècle :

Il n'est pas qu'en nos derniers troubles le party Catholic ne fust encores subdivisé en Politic, que l'on estimoit de pire condition que le Huguenot, parce qu'il plaidoit pour la paix, et le Ligueur. ${ }^{2}$

La complexité de la situation politico-religieuse au sein du royaume et les difficultés à instaurer une paix durable fragilisent le pouvoir royal. Henri III voit son autorité contestée par les catholiques intransigeants, les Guise en tête, sa capacité à gérer la situation étant remise en question. Espérant réduire un danger, il décide, en décembre 1588, de faire assassiner le duc de Guise et son frère, le cardinal de Lorraine. Il s'attire en fait la haine de toute la France ligueuse. Isolé, Henri III se rapproche du protestant Henri de Navarre afin de faire front commun contre la Ligue. Gouverneur général et protecteur des Églises réformées, Navarre est aussi héritier présomptif de la couronne de France selon la loi salique, Henri III n'ayant pas d'enfant et plus de frères. En août 1589, Henri III est assassiné par le moine ligueur Jacques Clément. Henri de Navarre devient Henri IV mais, non reconnu par la majorité catholique qui refuse un roi hérétique, il doit conquérir son royaume. La conversion du roi apparaît de plus en plus nécessaire pour soumettre l'ensemble des sujets à l'autorité du nouveau roi. Les Politiques œuvrent dans ce sens, convaincus qu'il s'agit là d'une étape essentielle pour restaurer la paix intérieure. Envisagé avec méfiance par la Ligue, redouté puis déploré par l'Union calviniste, le changement de religion du roi préoccupe autant les deux autres partis que la question plus générale de la coexistence. En juillet 1593, Henri IV abjure le protestantisme puis se convertit au catholicisme.

\section{Cadre théorique}

Afin de comprendre la relation entre ces discours et l'idéologie ${ }^{3}$ qui les sous-tend, il convient de définir quelques termes. Selon Van Dijk (2006 : 116-117), les idéologies sont des ensembles d'idées, des systèmes de croyances, de représentations, socialement partagées par les membres d'un groupe dont elles définissent l'identité, et ce, en relation avec d'autres groupes $^{4}$. Ces représentations ont un caractère fondamental, axiomatique : elles constituent la base des discours du groupe et contrôlent d'autres représentations de nature plus concrète, donnant à ces dernières une cohérence, facilitant leur acquisition et leur utilisation dans la vie quotidienne. Les idéologies indiquent aux membres du groupe les valeurs qui sont pertinentes et leur permettent d'organiser et de contrôler leurs actions en fonction des objectifs et intérêts $d u$ groupe. Insistant sur la relation entre un groupe et d'autres groupes dans sa définition de l'idéologie, Van Dijk (2006 : 124) distingue l'ingroup et l'outgroup. L'ingroup existe dans son opposition à tout ce qui est en dehors de lui, c'est-à-dire l'outgroup. Le discours de l'ingroup sur lui-même se caractérise par une tendance à valoriser ses aspects positifs et à relativiser ses aspects négatifs. À l'inverse, les aspects positifs de l'outgroup sont minimisés

\footnotetext{
${ }^{2}$ Cité dans Jouanna et al. (1998: 1212).

${ }^{3}$ Pour l'histoire du concept depuis les années 1960 et sa réactualisation par Van Dijk, voir l'article de Bonnafous $2002: 301-303$.

${ }^{4}$ Notre traduction de l'anglais en ce qui concerne les termes en italique présents dans ce paragraphe. Les termes ingroup/outgroup ont été conservés.
} 
alors que ses aspects négatifs sont exagérés ${ }^{5}$. Nous appuyant sur cette polarisation idéologique définie par Van Dijk, nous proposons d'ajouter un troisième groupe à ce schéma binaire. Ce troisième groupe correspond à un espace de transition dans lequel se trouvent ceux qu'il faut convaincre de rester dans ou de rejoindre l'ingroup.

L'étude des expressions référentielles a donné lieu à plusieurs conceptions. Considérant que le sujet parlant agit lors du processus de désignation, Apothéloz \& ReichlerBéguelin (1995) défendent une conception constructiviste de la référence. L'étude de l'évolution de la référence consiste à examiner « la façon dont les sujets parlants contrôlent, dans leurs productions langagières, l'évolution de ce que nous appelons les objets-dediscours » (Apothéloz et Reichler-Béguelin, 1995 : 2). Cette étude permet de mettre en évidence les stratégies discursives de l'auteur d'un discours. Celui-ci dispose en effet, "pour désigner un objet donné, d'une série non close d'expressions linguistiques utilisables à conditions référentielles égales. » (Apothéloz \& Reichler-Béguelin 1995 : 10). Les choix opérés par le locuteur lors des opérations de catégorisation et recatégorisation peuvent correspondre à différentes visées : argumentation, clarification, polyphonie, visée sociale (ménager la face de l'autre) ou encore visée esthétique-connotative. Les expressions référentielles anaphoriques peuvent réaliser les transformations subies par l'objet-de-discours (la transformation est opérée ou marquée par l'anaphorique), ou ignorer les transformations subies par l'objet-de-discours (omission d'attributs prédiqués), ou encore entériner les transformations subies par l'objet-de-discours (homologation d'attributs prédiqués) (Apothéloz \& Reichler-Béguelin 1995 : 12-13).

Examinant les catégorisations et recatégorisations dans deux discours, cette étude est qualitative. En ce qui concerne les anaphores, l'analyse porte essentiellement sur les anaphores à contenu lexical. Toutefois, certaines anaphores pronominales seront aussi étudiées.

\section{Catégorisation et recatégorisations dans la Declaration du Duc de Mayenne, pour la Reünion de tous les Catholiques de ce Royaume, décembre 1592 / 1593 (texte de la Ligue)}

La Declaration du Duc de Mayenne, pour la Reünion de tous les Catholiques de ce Royaume, est rédigée en décembre 1592, puis publiée par cri public et sous forme imprimée au cours de l'année 1593, avant la conversion du roi. Elle s'adresse à tous les catholiques du royaume. Décrivant la situation politico-religieuse dans le Royaume de France, elle fait le récit des événements récents alternant avec des séquences évoquant un passé plus ancien idéalisé et un futur souhaité ou redouté. La possible conversion du roi suscite l'inquiétude des ligueurs. Se fera-t-elle et si oui sera-t-elle authentique ou feinte, valable ou non valable ? L'objectif de la Declaration est de réconcilier les catholiques divisés afin de mener une lutte commune contre l'hérésie protestante.

\footnotetext{
5 « the underlying ingroup-outgroup polarization of ideologies: Our good things and Their bad things will tend to be emphasized, as is the case for the mitigation of Our bad things and Their good things. » Van Dijk (2006 : 124).
} 


\subsection{Les protestans}

Les protestants du royaume sont présentés par Mayenne comme une sous-catégorie du groupe plus large, dans l'espace et dans le temps, des hérétiques. L'objet-de-discours est introduit de façon non neutre et inclut d'emblée cet aspect religieux :

(1) ceux qui vouloyent introduire nouvelles sectes \& erreurs, contre la foy \& creance de leurs peres.

Le référent, en (1), ne correspond pas exclusivement aux protestants : dans le cotexte, Mayenne évoque la lutte des rois de France, au fil du temps, pour «conserver la Religion ». Les recatégorisations qui suivent cette première occurrence permettent de focaliser sur le groupe plus restreint des protestants du royaume en maintenant cette perspective religieuse (2) et (3).

(2) iusques à ces derniers temps que l'heresie s'est glissee si avant dans le Royaume, \& accreue par les moyens que chacun sçait

(3) les mauvaises impressions \& subtils artifices, dont les heretiques ont usé, pour leur persuader que ceste guerre n'estoit point pour la Religion

On observe, en (2), un glissement métonymique des individus agissant selon leurs convictions religieuses vers les idées qui les animent. La dénomination «les heretiques », donnée en (3), permet de désigner ce qui est présenté en (1) bien que le référent de (3) soit plus spécifique (les protestants pendant les guerres de religion). Les termes hérétiques et hérésie, caractérisant les protestants comme étant dans l'erreur du point de vue religieux, prédominent pour désigner ces derniers dans le texte.

Plusieurs recatégorisations métonymiques, gardant la perspective religieuse, sont présentes dans le texte, ainsi en (4), (5) et (6).

(4) une nouvelle doctrine

(5) leur Religion

(6) le mal

Les termes utilisés sont axiologiquement marqués (vérité vs erreur (2), bien vs mal (6)) et entérinent le schisme en (4) et (5). Cette façon de désigner l'outgroup par son système de croyances, et non par ses membres, permet de dissocier individus et idées dans un contexte où la conversion, qu'elle concerne le roi ou ses sujets, reste toujours possible.

Certaines recatégorisations sont faites dans une perspective plus politico-militaire. On observe ainsi une fragmentation du groupe des protestants mettant en avant son organisation politico-militaire : ses chefs (7), sa capacité militaire (8), ses assemblées politiques (9).

(7) le Roy de Navarre \& Prince de Condé

(8) ayant mandé les siens, assembla par leur prompte obeyssance une grande armée

(9) leurs conseils

Le roi de Navarre - futur Henri IV - et le prince de Condé (7) sont deux figures importantes du protestantisme dans le royaume. Chefs militaires, ils ont participé à plusieurs guerres contre les armées catholiques. Navarre et Condé sont ici associés dans un passage où 
Mayenne évoque la tentative d'Henri III de les ramener au catholicisme lors des «Estats generaux assemblez à Bloys " à la fin de l'année 1576. Condé était le chef, gouverneur général et Protecteur des églises réformées depuis 1574, avant d'être remplacé par Navarre au cours de l'année 1576. Cette année est marquée par la reprise du conflit.

Le groupe des protestants est présenté comme une puissance militaire capable de lever une armée redoutable (8) sur commandement de son chef. Mayenne relate ici la conquête militaire entreprise par Henri IV au cours des années 1589-1593.

\subsection{Les catholiques}

Les catholiques se subdivisent en plusieurs groupes, plusieurs instances, au sein du royaume et à l'extérieur de celui-ci. Nous analysons ici la façon dont la Ligue, les Politiques et les catholiques en général sont désignés dans le texte de Mayenne.

La Ligue, ou Sainte Union, a pour chef le duc de Mayenne. Celui-ci commence son discours en se mentionnant à la troisième personne :

(10) Charles de Lorraine, Duc de Mayenne, Lieutenant general de l'Estat \& Couronne de France

Mayenne n'utilise jamais le pronom je pour se désigner dans le texte. Il y a par contre de nombreuses occurrences du pronom nous dont plusieurs ont pour référent soit Mayenne lui-même, soit les ligueurs, selon différents degrés de métonymisation allant de la garde rapprochée à l'ensemble des ligueurs. Pour le chef de la Ligue, utiliser nous plutôt que je permet d'imprimer à son discours la force du nombre et d'éviter de donner l'impression que les idées soutenues émanent d'un seul homme. Le pronom nous peut donc être considéré comme une recatégorisation métonymique permettant un glissement du chef vers son groupe (11). Ce nous est d'ailleurs recatégorisé avec une dénomination précisant le référent (12).

(11) nous [la Ligue]

(12) les Catholiques unis

Le groupe des ligueurs subit lui-même des recatégorisations par fragmentation :

(13) un seul homme

(14) les morts

(15) Paris $[\ldots]$ assiegez

(16) les Princes unis pour la defense de la Religion

(17) tous les autres de ce party

(18) [les] Princes, Pairs de France, Prelats, Seigneurs, Gentilz-hommes, villes \& communautez, qui se sont unis ensemble pour la conservation de leur Religion.

Le référent de (13) correspond au régicide Jacques Clément. Mayenne évoque l'assassinat d'Henri III en isolant son auteur au sein de l'ingroup des ligueurs : « la main d'un 
seul homme ${ }^{6}$, sans l'ayde ny le sceu de ceux qui n'avoyent que trop d'occasion de la desirer ». Ne cachant pas une certaine satisfaction face à la mort du roi, il disculpe cependant l'ingroup de cette responsabilité ${ }^{7}$. Les morts en (14) correspondent au duc de Guise, ancien chef de la Ligue, et au cardinal de Lorraine, tous deux frères du duc de Mayenne, assassinés sur ordre d'Henri III en 1588. Ce double assassinat déchaîna la France ligueuse contre le roi. Paris assiégé (15) désigne, par métonymie, le noyau irréductible des ligueurs. Les recatégorisations en (16), (17) et (18) permettent à Mayenne de montrer que les membres de l'ingroup appartiennent à toutes les catégories de la société et qu'ils sont tous animés par le même désir de défendre « la Religion $»^{8}$.

Dans son discours, Mayenne désigne aussi les Politiques, les catholiques modérés, sans toutefois utiliser ces termes. L'objet-de-discours est introduit de façon négative :

(19) [les] auteurs du plus meschant, desloyal, \& pernicieux conseil, qui fut jamais donné à Prince

Les recatégorisations opèrent une fragmentation chronologique (20), (21) ou professionnelle (22) et marquent l'appartenance religieuse. Toutefois, le développement métalinguistique en (22) introduit un doute quant à l'appartenance des Politiques au groupe des catholiques.

(20) plusieurs Catholiques pres du feu Roy [Henri III]

(21) les Catholiques qui l'assistent [Henri IV]

(22) [des] Magistrats qui s'attribuent le nom de Catholiques

Parallèlement aux deux catégorisations (Ligueurs et Politiques), l'auteur présente les catholiques de façon plus globale transcendant les limites chronologiques et géographiques :

(23) Les Catholiques

Ce groupe idéalisé, souhaité comme homogène et éternel, est recatégorisé par une fragmentation qui prend en compte différentes étapes de son histoire (24), (25). Cet idéal est

\footnotetext{
${ }^{6}$ Cette distanciation par rapport à Jacques Clément, qui n'est pas mentionné par son nom, contraste avec le portrait dont bénéficie le régicide dans les libelles ligueurs produits peu après l'assassinat du roi. Pour cette question, voir Goderniaux (2020) : l'auteur examine la répétition de lieux communs comme stratégie rhétorique et constate notamment la répétition, dans plusieurs libelles, de la comparaison de Jacques Clément à la figure vétérotestamentaire de Judith tuant Holopherne.

${ }^{7}$ Cette construction rhétorique dans laquelle Mayenne s'attache à innocenter le groupe lui permet de bénéficier de cette disculpation sans avoir à faire la démarche pour sa propre personne de façon explicite. Mayenne fut en effet soupçonné d'avoir favorisé l'assassinat du roi, par non-empêchement ou par instigation (Boucher 1998 : 1090-1091). Dans ses travaux sur les différentes versions du De justa Henrici tertii abdicatione, Zwierlein (2016 : 180) rappelle comment, dès le printemps 1589, l'idée de tyrannicide appliquée à Henri III prend place dans le discours de la Ligue, et souligne à quel point ce discours contribue à légitimer l'action du groupe en même temps qu'il permet une prise de conscience constitutionnelle. Ainsi, le discours de 1592/1593 témoigne-til d'un réajustement par rapport à celui de 1589. Par ces formulations (« un seul homme », «ceux qui...»), Mayenne réussit donc tout à la fois à masquer sa tentative d'auto-disculpation, à mettre en avant la force du groupe et à présenter l'assassinat du roi comme un acte isolé.

${ }^{8}$ Signalons que la Ligue se subdivise elle-même en différentes tendances dont la plus zélée finit par être condamnée par Mayenne (pour cette question voir Jouanna et al. (1998 : 357-359)). Toutefois, Mayenne n'a pas intérêt à souligner ces divisions internes dans son texte.
} 
réaffirmé à la fin du discours par fusion des différents sous-groupes redéfinissant les protagonistes de façon binaire et axiologiquement marquée (26).

(24) [les Catholiques] que l'union de l'Eglise devoit inseparablement conjoindre, se sont $[\ldots]$ armez les uns contre les autres

(25) Lors que les Catholiques n'estoyent encore divisez à la defense de leur religion

(26) Les Catholiques ensemble contre les heretiques

\subsection{Le roi}

La légitimité d'Henri de Navarre / Henri IV comme roi de France est contestée. Dans son discours, le Duc de Mayenne opère une distinction entre la personne incarnant la fonction royale et la dignité royale elle-même, empreinte d'une longue tradition valorisée par les ligueurs.

Pour désigner Henri de Navarre, Mayenne n'utilise jamais le terme «Henri IV », manifestant ainsi qu'il ne le reconnaît pas comme roi de France. L'objet-de-discours est introduit par le recours à un autre titre qui ne lui est pas contesté :

\section{(27) Le Roy de Navarre}

Henri est roi de Navarre depuis 1572. Cependant, dans le cotexte de (27), Mayenne évoque les quelques mois ayant suivi l'assassinat d'Henri III en août 1589. Henri de Navarre était donc, à partir de cette date et selon la loi salique, roi de France.

Dans les recatégorisations qui suivent, la relation entre Navarre et la couronne de France est problématisée. Relatant des événements passés, Mayenne indique les prétentions de Navarre à la couronne comme héritier potentiel lors du règne d'Henri III.

(28) le Prince qui se pretendoit appellé par les loix à la couronne

L'expression «les loix » renvoie aux lois fondamentales du royaume qui, par leur ancienneté, sont considérées comme gardiennes de la monarchie française. Dans un long développement sur ces lois, Mayenne insiste sur la nécessité d'être catholique pour devenir roi de France : «la Loy fondamentale du Royaume, qui requiert au Prince qui pretend droict a la Couronne, avec la proximité du sang, qu'il soit Catholique ». La «proximité du sang» n'est donc pas considérée comme une condition suffisante.

D'autres recatégorisations envisagent Navarre comme roi de France de façon hypothétique dans un cotexte analysant le présent (après 1589). La dimension religieuse est mise en avant et axiologiquement marquée (29) et le risque pour les catholiques de ne pas pouvoir contester les décisions de ce roi hypothétique est évoqué (30).

(29) un Roy heretique

(30) Maistre absolu

Certaines recatégorisations indiquent des qualités politico-militaires avérées et déplorées (31), (32), même si, là encore, le terme hérésie est préféré à celui des églises réformées revendiqué par les protestants. 
(31) Maistre de la campaigne

(32) Chef \& protecteur de l'heresie

Ne reconnaissant pas Navarre comme roi de France, Mayenne n'en manifeste pas moins de respect pour la couronne de France et la succession des rois depuis Clovis. Cet objet-de-discours est introduit en insistant sur la dimension religieuse : les rois de France ont toujours été catholiques (33).

(33) Noz Roys du nom de Tres-Chrestiens \& premiers enfans de l’Eglise

Les recatégorisations qui suivent permettent de fragmenter cette lignée, indiquant les différents mérites des rois de France sur le plan religieux (34), (35), ou de reformuler la catégorisation initiale en insistant sur la dimension chronologique (36).

(34) Les uns pour acquerir ce tiltre si glorieux [...]

(35) Les autres combatu plusieurs fois [...]

(36) La succession de tant de Roys, depuis Clovis jusques à present

Certaines recatégorisations reconnaissent le statut de roi de France à Henri III (37) et au cardinal de Bourbon (38).

(37) le feu Roy

(38) Recogneu pour Roy Monseineur le Cardinal de Bourbon

Le traitement accordé à Henri III (37) paraît neutre comparé au déferlement de violence verbale dont le dernier des Valois a fait l'objet dans les prédications parisiennes et les libelles de la Ligue après l'assassinat des Guise en 1588 et jusqu'à son propre assassinat en $1589^{9}$. Ainsi, moins de quatre années plus tard, Mayenne cherche à recentrer le discours ligueur sur sa nouvelle cible, à savoir Henri de Navarre. Afin de donner plus de poids à cet ajustement, les griefs autrefois exprimés contre Henri III sont ici mis de côté et son statut royal ne lui est pas contesté contrairement à Navarre.

Prince du sang et oncle de Navarre, le cardinal de Bourbon, catholique, est imposé par la Ligue comme héritier légitime d'Henri III. Il est reconnu comme roi par la Ligue en 1589 et proclamé comme tel par le parlement de Paris en 1590. Il meurt peu de temps après.

\section{Catégorisation et recatégorisations dans la Lettre au Roy, 1593 (texte protestant)}

La Lettre au Roy est une œuvre collective rédigée très probablement par Philippe Duplessis-Mornay ${ }^{10}$ au nom des assemblées politiques des réformés après juillet 1593. Elle dresse un tableau de la situation des protestants en France selon plusieurs points de vue et fournit un bilan des quatre années de règne d'Henri IV. Les auteurs font le constat que, malgré

\footnotetext{
9 Voir notamment Jouanna et al. (1998 : 351-354). Goderniaux (2020) souligne le «processus de désacralisation » du roi à l'œuvre dans les libelles produits après l'assassinat des Guise. Ainsi Henri III est-il désigné par l'expression «vilain Herodes » (anagramme d'Henri de Valois) qui, répétée d'un libelle à l'autre, contribue à ôter au souverain sa dignité royale.

${ }^{10}$ Pour l'attribution de ce texte à Duplessis-Mornay, ainsi que son contexte historique, voir Daussy $(1998: 212)$ et Fornerod (1998: 227).
} 
les nombreux services rendus au nouveau roi, le statut des réformés ne s'est pas amélioré. Ils réitèrent donc leurs requêtes. La conversion du roi suscite l'inquiétude des protestants. Devenu officiellement catholique, le roi a-t-il l'intention de défendre leurs intérêts et est-il en mesure de le faire ? L'objectif de la Lettre est de faire pression sur le roi pour qu'il agisse en faveur des protestants.

\subsection{Les protestans}

Les réformés du royaume sont présentés comme un groupe relativement homogène. L'objet-de-discours est introduit de façon non neutre et inclut d'emblée les aspects politique et religieux :

(39) vos tres humbles subjects de la relligion reformee

Certaines recatégorisations sont faites dans une perspective plus politique distinguant deux périodes chronologiques : les protestants soutenant Henri dans la tourmente (jusqu'à 1593) (40) (41) (42) et les protestants sujets du roi établi (43) (44) (45). Les termes utilisés insistent sur les services rendus, le sens du sacrifice et la loyauté des protestants. L'utilisation du terme sujet (39) (43) (45) permet d'une part de reconnaître pleinement à Henri IV son statut de roi, et d'autre part d'inscrire les protestants comme faisant partie intégrante du royaume.

(40) ceulx qui espandoient leur sang si librement pour vous

(41) celui qui vous deffendoit jadis

(42) vos bons serviteurs

(43) vos povres subjects

(44) les plus loyaulx François

(45) la plus sincere partie de vos subjects

L'utilisation du terme huguenot dans une recatégorisation (46) produit un effet polyphonique. Utilisé pour désigner les réfomés de façon péjorative (évocation de leur tendance à se révolter contre l'autorité) ${ }^{11}$, ce terme passe peu à peu dans le langage courant. Il contraste ici avec les autres expressions utilisées.

(46) les huguenots

D'autres recatégorisations offrent une perspective plus religieuse : les protestants sont présentés selon eux-mêmes (47) ou, de façon polyphonique, selon les catholiques (48). En (49), le terme sujets de la religion sous-entend religion réformée. Il peut aussi renvoyer au terme religion prétendue réformée utilisé par les catholiques pour désigner officiellement et péjorativement les protestants du royaume.

(47) les plus chrestiens

(48) heretiques

\footnotetext{
${ }^{11}$ Pour l'histoire de ce mot, voir Jouanna et al. (1998 : 983).
} 
(49) vos dicts tres humbles subjects de la relligion ${ }^{12}$

Ces recatégorisations, qu'elles aient une orientation politique ou religieuse, contribuent au discours de valorisation de l'ingroup par une mise en relief de différentes qualités (40) (41) (42) (44) (45) (47). L'outgroup est quant à lui indirectement dévalorisé : les catholiques ne sont donc ni les plus loyaux français (44) (45) ${ }^{13}$, ni les plus chrétiens (47). Les souffrances ressenties par les protestants (43) et le sentiment d'injustice quant à l'utilisation de certains termes les désignant (46) (48), contribuent également à dévaloriser l'outgroup, source de tous ces maux.

On observe par ailleurs quelques fragmentations reflétant une construction rhétorique destinée à mettre en scène le rôle de représentation de ce discours ${ }^{14}$ tout en montrant que, audelà de la diversité, les différentes voix convergent vers la même vérité. Ces recatégorisations métonymiques ont une valeur généralisante et permettent de jouer sur le pathos en exprimant l'intensité des sentiments de façon graduelle (50) (51).

(50) Le vulgaire dict là-dessus (car il ne voit pas plus avant), si c'est de franche volonté, qu'attendons nous plus de son affection?

(51) Certes, sire, les plus advisés estiment qu'il est impossible que vostre majesté oublie les graces qu'elle a receues de Dieu

La Lettre comporte également des recatégorisations dialogales. Les protestants sont généralement présentés à la troisième personne. Cependant les auteurs leur donnent parfois la parole dans une forme de discours direct (passage de ils à nous) (52) ou s'incluent dans le groupe des protestants renonçant temporairement à la distanciation objective du médiateur (nous incluant les auteurs) (53).

(52) Mais tout de mesmes ils discourent, sire : si au milieu de ses prospérités, il nous a mescogneus

(53) Qui ne voit que le premier project estoit et contre vous, et contre nous ? Vous qu'ils ont contrainct en vostre conscience, qu'ils pretendent par degrés animer contre nous?

\subsection{Les catholiques}

Nous analysons ici la façon dont les catholiques sont présentés dans ce discours protestant : la Ligue, les Politiques, les catholiques en général.

La Ligue est désignée par ce terme dans la Lettre. L'objet-de-discours est introduit en association avec le qualificatif tyrannique.

(54) Les tyranniques edicts de la Ligue

Evoquer la tyrannie n'est pas neutre et constitue un écho aux théories monarchomaques contestant l'absolutisme royal au cours de la seconde moitié du XVIème

\footnotetext{
${ }^{12}$ Nous mettons en gras ces termes. Il en est de même dans les exemples qui suivent.

${ }^{13}$ Ces termes, utilisés par les protestants pour mettre en évidence leurs qualités, résonnent d'une façon particulière dans un contexte où le royaume de France est réellement menacé par l'ingérence des puissances étrangères et où ultra-catholiques et protestants s'accusent réciproquement d'association dangereuse avec cellesci.

${ }^{14}$ Rappelons que ce texte émane des assemblées politiques des réformés.
} 
siècle $^{15}$. Initiées par des auteurs protestants sous les rois catholiques, ces théories connaissent un développement catholique lorsque Navarre devient héritier présomptif puis roi de France. Être qualifié de tyran est stigmatisant et peut avoir des conséquences extrêmes comme en témoigne l'assassinat d'Henri III. Dans la Lettre au Roy, la présence de ce terme, même s'il n'est pas associé à Henri IV, produit un effet auquel le destinataire est forcément sensible.

Les recatégorisations concernant la Ligue permettent de mettre en scène les individus derrière le groupe (spécification) (55), et de focaliser sur son bastion irréductible (56) ou son chef (57) (fragmentation). En (58), la recatégorisation rappelle clairement à Henri IV où se situe la Ligue sur l'échiquier politico-militaire.

(55) Pour feu de joie de la paix avec ceulx de la Ligue, on vous aura faict brusler vos bons subjects

(56) Paris

(57) Le duc de Mayenne

(58) Vos ennemis

Les Politiques, les catholiques modérés, sont catégorisés par leur attitude à l'égard du roi qui contraste avec celle de la Ligue :

(59) les catholiques romains qui suivent vostre majesté

Les recatégorisations donnent toutefois l'impression d'un groupe mal identifié (indétermination) (60) mais dont l'influence empiète sur la clairvoyance (61) et l'autorité du roi (62) (63).

(60) Voyés, sire, par quels degrés on vous a mené à la messe.

(61) Les plus fins vous faisoient croire

(62) Ceste conference a commencé par ung corps qui ne s'auctorisoit poinct de vous

(63) ceulx qui vous possèdent

Parmi les recatégorisations, on observe parfois une fusion des objets-de-discours tendant à faire des catholiques une nébuleuse dont les relations internes sont imprécises, incertaines, suscitent le doute et l'inquiétude chez les protestants :

(64) N'attendons que mal, puisque nostre mal est en puissance d'aultrui

\subsection{Le roi}

Dans la Lettre, la mise en scène du roi se caractérise par une alternance entre termes d'adresse et désignatifs. L'objet-de-discours est introduit par un terme d'adresse :

(65) Sire

Dans les nombreuses recatégorisations qui suivent, le référent est balloté entre différents statuts : passés, présents, hypothétiques (souhaités ou redoutés).

\footnotetext{
${ }^{15}$ Pour cette question, voir Jouanna et al. (1998 : 1109-1111).
} 
Certaines recatégorisations insistent sur le statut de roi et la fonction royale témoignant ainsi de la déférence des protestants vis-à-vis de leur souverain :

(66) vostre majesté

(67) roy

(68) leur prince naturel

(69) celui que Dieu avoit, par la protection de son Eglise, amené à la succession de ce royaulme

D'autres recatégorisations rappellent à Henri IV son statut avéré de protecteur dans des tournures donnant à ce titre un niveau de prestige semblable à celui de roi :

(70) Qu'ayans cest honneur de se voir pour roy celui qu'ils avoient eu l'honneur d'avoir pour protecteur ; et en auctorité d'interiner leurs requestes, celui qui auroit eu le zele au milieu de tant de dangers de les presenter.

Plusieurs recatégorisations permettent aux auteurs de la Lettre de souligner les dérives, aux yeux des protestants, des choix politico-religieux d'Henri IV :

(71) Car la trefve [...] apres mesme vostre pretendeue conversion qui vous debvoit faire roy, vous a elle pas reduict à estre chef de parti ? [...] de roy par la trefve, vous soyés veneu chef de parti; de chef de parti, par ceste paix, vous deveniés leur capitaine general contre les huguenots?

En (71), un double effet polyphonique tend à invalider certains changements de statut : d'une part la conversion veut être envisagée comme prétendue par les protestants, et d'autre part, si la conversion était considérée comme nécessaire par les catholiques pour accéder pleinement - au statut de roi, ce n'était pas le cas pour les protestants qui ont reconnu Navarre comme leur nouveau souverain dès la mort d'Henri III. Cette conversion semble même enlever au roi mentionné dans cette séquence son authenticité. Dans les recatégorisations suivantes, l'objet-de-discours perd ce statut pour redescendre au niveau de chef de parti puis de capitaine général menaçant politiquement puis militairement les protestants. La séquence est toutefois modalisée par la forme interrogative et l'emploi du subjonctif permettant de placer le destinataire de la Lettre dans une position instable l'incitant à faire des choix que les protestants espèrent être en leur faveur. Le roi se trouve donc entre l'ingroup et l'outgroup, dans cet espace de transition où tout peut basculer, vers le meilleur comme vers le pire.

La crainte de ces dérives suscite des recatégorisations insistant sur le statut souhaité de protecteur. En (72), les auteurs évoquent le risque auquel s'expose Henri IV de voir les protestants se tourner vers un autre protecteur s'il ne se montre pas capable de continuer luimême cette mission. La Lettre est conclue en (73) par une série de recatégorisations conciliant les deux statuts de roi et de protecteur, invitant clairement Henri IV à rejoindre l'ingroup.

(72) Sire, voulés vous bien leur oster l'envie d'un protecteur ? Ostés en la necessité ; soyés le donc vous mesmes ; continués dessus eulx ce premier soin, ceste premiere affection.

(73) En estre, sans aultres deputés, et avec plus de gré, le juge, si vous voulés, et l'advocat, l'impetrant et l'octroyant ensemble. 


\section{Conclusion}

Le trouble suscité par la situation politico-religieuse du royaume et l'inquiétude quant aux conséquences des choix religieux du roi transparaissent dans les deux textes étudiés. La mise en scène des différents acteurs (les protestants, les catholiques, le roi) et la perspective adoptée varient cependant entre les deux discours.

Le groupe des protestants est présenté de façon homogène dans chacun des deux textes. Dans la Declaration de Mayenne (la Ligue), la perspective est avant tout religieuse : les protestants sont assimilés au groupe plus large des hérétiques, selon le principe de dévalorisation de l'outgroup. Dans la Lettre au Roy, les protestants sont mis en scène comme un groupe uni. Les quelques fragmentations ont soit une valeur généralisante, soit une fonction de hiérarchisation de l'intensité des sentiments n'indiquant en rien d'éventuelles dissensions internes (religieuses ou politiques).

Les catholiques se subdivisent en plusieurs groupes et sont présentés ainsi dans les deux textes. Dans la Declaration, les ligueurs et les politiques apparaissent comme deux groupes distincts. Le groupe des catholiques en général se dessine aussi dans le discours : les recatégorisations évoquent les divisions déplorées et la réunion souhaitée. Les catholiques non ligueurs se trouvent donc dans cet espace de transition entre l'ingroup et l'outgroup. L'objectif de Mayenne est de les convaincre de rejoindre l'ingroup. Dans la Lettre au Roy, les différents groupes catholiques de l'outgroup sont distingués. On observe cependant quelques fusions des objets-de-discours tendant à faire des catholiques une nébuleuse redoutable pour les protestants.

La personne du roi et la dignité royale sont distinguées de façon subtile dans les deux textes. Dans la Declaration, Mayenne insiste sur le critère de catholicité pour accéder à la couronne, celui du sang n'étant pas considéré comme suffisant. Navarre n'est donc pas présenté comme roi de France ou seulement de façon hypothétique, dans une perspective monarchomaque brandissant le spectre d'un absolutisme délétère pour la religion catholique. La fonction royale est cependant respectée, valorisée par la succession des rois de France qui, depuis Clovis, ont toujours lutté contre l'hérésie et pour la défense de la Religion. Dans la Lettre au Roy, la conversion de Navarre est présentée comme un tournant décisif dans un processus défavorable aux protestants. Le statut de roi n'est pas contesté. Cependant, plusieurs recatégorisations insistent sur le statut avéré et souhaité de protecteur élevant cette fonction au même niveau que la fonction royale. Dans ce texte, c'est le roi qui est placé dans l'espace de transition entre l'ingroup et l'outgroup. L'objectif des auteurs est de convaincre Henri IV de rester dans l'ingroup même s'il semble avoir rejoint l'outgroup.

Ces deux textes reflètent deux perspectives différentes. La Declaration du chef de la Ligue se caractérise par un discours dans lequel la dimension religieuse prédomine. Dans la Lettre au Roy, le discours reflète la stratégie protestante préférant stigmatiser l'opposition ingroup-outgroup en termes politiques.

La conversion du roi constitue une étape essentielle dans l'entreprise des Politiques pour lesquels seul un pouvoir royal fort et la paix intérieure peuvent garantir l'intégrité du royaume de France face aux puissances étrangères. Contribuant à la chute des derniers bastions ligueurs, la conversion ne résout cependant pas tous les problèmes et laisse les catholiques les plus intransigeants dans l'insatisfaction et les réformés du royaume dans l'incertitude et l'insécurité. C'est au cours des années suivantes, alors que la paix se construit difficilement, que l'édit de Nantes est élaboré. Signé en 1598, l'édit instaure un régime de 
coexistence confessionnelle original qui entend réunir tous les sujets du royaume, plaçant l'intérêt de l'État au-dessus de la religion.

\section{Bibliographie}

Apothéloz, Denis, et Reichler-Béguelin, Marie-José (1995), «Construction de la référence et stratégies de désignation. » Travaux Neuchâtelois de Linguistique (TRANEL), Institut des sciences du langage et de la communication (Neuchâtel, Suisse), no. 23: p. 227271. <hal-00869154> https://hal.archives-ouvertes.fr/hal-00869154/document

Bonnafous, Simone (2002), «Idéologie. » in Patrick Charaudeau et Dominique Maingueneau (éds), Dictionnaire d'analyse du discours, Paris, Éditions du Seuil, p. 300-303.

Boucher, Jacqueline (1998), « Mayenne » in Jouanna et al. (éds), Histoire et dictionnaire des Guerres de religion, Paris, Robert Laffont, p. 1088-1092.

Daussy, Hugues (1998), «Au cœur des négociations pour l'édit de Nantes : le rôle de Philippe Duplessis-Mornay. » in Michel Grandjean et Bernard Roussel (éds), Coexister dans l'intolérance. L'édit de Nantes (1598), Genève, Labor et Fides, p. 207-224.

Fornerod, Nicolas (1998), «L'édit de Nantes et le problème de la coexistence confessionnelle dans la pensée de Philippe Duplessis-Mornay » in Michel Grandjean et Bernard Roussel (éds), Coexister dans l'intolérance. L'édit de Nantes (1598), Genève, Labor et Fides, p. 225-252.

Goderniaux, Alexandre (2020) (à paraître), «Répéter pour convaincre. Usages polémiques de la répétition dans les libelles de la Ligue parisienne (1585-1594). » in Loula AbdElrazak et Valérie Dusaillant-Fernandes (éds), La Répétition du Moyen Âge à nos jours, Bruxelles, Peter Lang.

Jouanna, Arlette, Boucher, Jacqueline, Biloghi, Dominique et Le Thiec Guy (1998), Histoire et dictionnaire des Guerres de religion, Paris, Robert Laffont.

Van Dijk, Teun A. (2006), «Ideology and discourse analysis. » Journal of Political Ideologies 11(2), p. 115-140.

Zwierlein, Cornel (2016), The Political Thought of the French League and Rome (15851589), Genève, Droz.

\section{Corpus}

Declaration du Duc de Mayenne, pour la Reünion de tous les Catholiques de ce Royaume (1592).

Le texte fut rédigé en 1592. La publication utilisée ici date de 1593. Le titre exact est :

Declaration faicte par Monseigneur le Duc de Mayenne Lieutenant general de l'Estat \& Couronne de France, pour la Reünion de tous les Catholiques de ce Royaume, A Lyon, Par Jean Pillehotte, Libraire de la saincte Union, 1593.

L'exemplaire utilisé pour cette étude est diponible en ligne sur Gallica :

https://gallica.bnf.fr/ark:/12148/bpt6k798552/f1.image

Lettre au Roy (1593) : œuvre collective, rédigée par Philippe Duplessis-Mornay au nom des assemblées politiques des réformés. 
Le texte manuscrit a été publié dans les Mémoires et Correspondance de DuplessisMornay, tome cinquième, A Paris, Chez Treuttel et Würtz, 1824 (p. 535-544). Cette édition est disponible en ligne :

https://archive.org/details/mmoiresetcorres06morngoog/page/n9 